\title{
Development of Myasthenia Gravis in Systemic Lupus Erythematosus
}

$\underline{\text { Nano Kigawa }^{a}}$, Christian Pineau ${ }^{b}$, Ann Clarke ${ }^{c, d}$, Emil Nashi E ${ }^{d}$, Évelyne Vinet ${ }^{c, b}$, Martin Veilleux Sasha Bernatsky ${ }^{c, b}$

${ }^{a}$ McGill University Health Centre, Montreal, Canada

${ }^{b}$ Division of Rheumatology, McGill University Health Centre, Montreal, Canada

${ }^{c}$ Division of Clinical Epidemiology, McGill University Health Centre, Montreal, Canada

${ }^{\mathrm{d}}$ Division of Allergy and Immunology, McGill University Health Centre, Montreal, Canada

e Department of Neurology and Neurosurgery, Montreal Neurological Institute, McGill University, Montreal, Canada

\section{Abstract}

Objectives: To present a case report and perform a literature review, as well as estimate myasthenia gravis (MG) incidence in a systemic lupus erythematosus (SLE) cohort.

Materials and methods: For the literature review, we searched MEDLINE and PubMed for case reports of SLE and MG. We also calculated MG incidence within our clinical SLE cohort (females only).

Results: Eleven articles met our criteria, providing 13 SLE patients who developed MG. The majority were female (84.6\%), with the average ages 25.6 and 33.5 years at diagnoses of SLE and MG, respectively. In 380 SLE female patients followed for 2850 person-years, one MG case occurred.

Conclusion: MG in SLE is a rare event.

Keywords: Myasthenia gravis, lupus erythematosus, systemic, incidence rate

Received: $17 / 12 / 2013$

Accepted: $14 / 01 / 2014$

Published: 20/02/2014

How to cite this article: Kigawa N, Pineau C, Clarke AE, Nashi E, Vinet É, Veilleux M, Bernatsky S. Development of Myasthenia Gravis in Systemic Lupus Erythematosus, EJCRIM 2014;1:doi: 10.12890/2014_000020

Conflicts of Interests: The authors declare that they have no conflicts of interest in this research.

This article is licensed under a Creative Commons Attribution Non-Commercial 4.0 License 


\section{Introduction}

We recently treated a patient with systemic lupus erythematosus (SLE) who presented with diplopia and weakness suggestive of myasthenia gravis (MG), 26 years after SLE diagnosis. Her initial SLE features were malar rash, photosensitivity, oral ulcers, arthritis, pleuritis, seizures, lymphopenia, and anti-dsDNA, antiphospholipid and antinuclear antibody (ANA) positivity. Although the MG symptoms were characteristic (ptosis, dysphagia, dysphonia and muscle weakness) and the patient initially seemed to respond to pyridostigmine bromide, the diagnosis was ultimately rejected in the face of repeatedly negative acetylcholine receptor (AChR) antibodies and non-confirmatory electrodiagnostic studies. This prompted a systematic review and synthesis of existing literature on the topic.

MG and SLE are both autoimmune diseases, occurring more often in women than in men.[1-4] Both conditions have their highest prevalence at reproductive age and beyond and are characterised by autoantibodies (including ANA, which can be present in both).[2-4]

MG is an autoimmune neuromuscular disease caused by the presence of autoantibodies mainly directed against the $\alpha$-subunit of the AChR[4], but also muscle-specific tyrosine kinase (MuSK). $[1,3,4]$

Although coexistence of these two diseases has been documented in case reports [1-4], the actual incidence rate of MG in SLE has never been estimated. In this study, we perform a literature review of published cases, to describe demographic and clinical characteristics, and present our estimate of the incidence of MG in a clinical cohort of female SLE patients.

\section{Methods}

A systematic literature search was conducted for articles published between 1994 and 2013, using MEDLINE and PubMed terms "myasthenia gravis" AND "lupus erythematosus, systemic". The titles and abstracts of the search results were reviewed. Articles in English, French, Spanish and Japanese were reviewed and both adults and paediatric patients were considered. Articles were included in our analyses if they reported a case of SLE with later onset of MG. We did not include, in our literature review, cases of SLE that followed a diagnosis of MG.

For the cases retrieved from the literature review, we compiled descriptive statistics of demographic (age, sex, race/ethnicity) and clinical characteristics (SLE duration and American College of Rheumatology (ACR) criteria, where available). We then calculated the incidence of confirmed MG in the female SLE cohort followed from 2004 to 2013 at the McGill University Health Centre Lupus Clinic. Each patient attends a clinical research visit once a year, as well as receiving interval care from the lupus specialists who work in the clinic. Classification criteria for MG are unclear, so we included only MG that had been clinically confirmed by a neurologist. We calculated 


\section{European Journal \\ of Case Reports in \\ Internal Medicine}

the MG rate in only female SLE patients only, because a minority $(<10 \%)$ of our cohort were male.

\section{Results}

\section{Literature review}

A literature search was conducted on MEDLINE and PubMed using the terms "myasthenia gravis" AND "lupus erythematosus, systemic", limited to the period of 1994-2013 and articles published in English, French, Spanish or Japanese. This resulted in 35 articles from MEDLINE and 31 from PubMed, but on cross-reference there were only 38 unique articles obtained from these searches (Fig. 1).

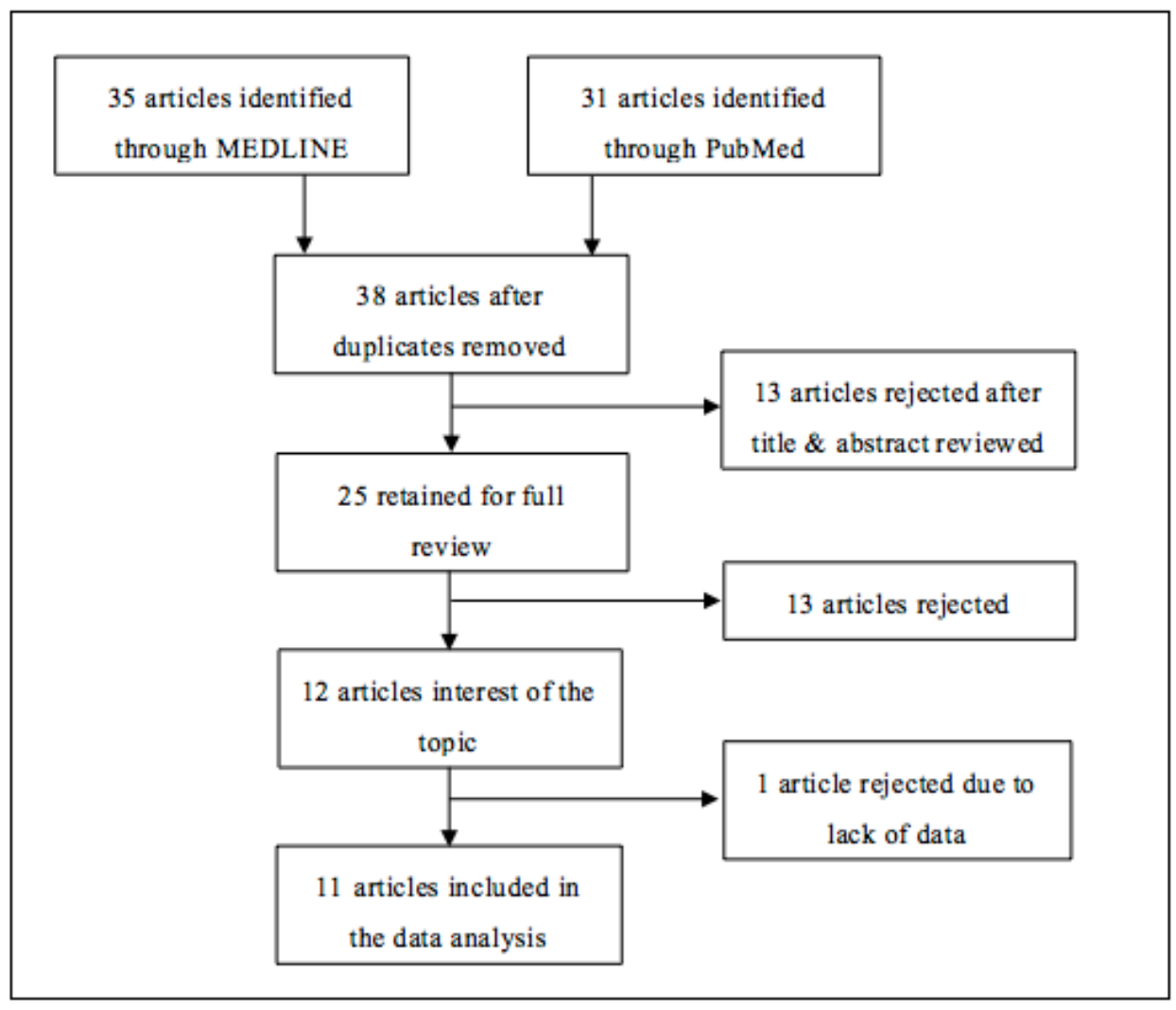

Figure 1. Results of the literature research

The titles and abstracts of these 38 articles were screened; 25 met our inclusion criteria and were retained for detailed review. The rejected papers focused only on one or the other of the diseases and/or both diseases were mentioned in the abstract or title, but they did not occur at the same time. After reviewing the 25 full papers that met our initial criteria, we retained only the 12 articles that reported at least one case of MG developing in established SLE. One article including 8 such cases was rejected after full review because there was no detailed information on demographics or 


\section{European Journal \\ of Case Reports in \\ Internal Medicine}

clinical factors; thus it was fully reviewed but not included in the analysis ${ }^{1}$. Consequently, 13 cases from 11 articles were analysed.

The resulting statistics calculated from the 13 cases in these 11 articles are shown in Table 1.

\begin{tabular}{|c|c|}
\hline Total $n$ & 13 \\
\hline Female, $\mathrm{n}(\%)$ & $11(84.6 \%)$ \\
\hline Age at SLE onset, mean $\pm S D$ (years) & $25.6 \pm 9.5$ \\
\hline Age at MG onset, mean $\pm S D$ (years) & $33.5 \pm 13.7$ \\
\hline SLE Duration*, mean $\pm S D$ (years) & $8.1 \pm 8.2$ \\
\hline \multicolumn{2}{|l|}{ Race/ethnicity, n (\%) } \\
\hline Caucasian & $1(7.7 \%)$ \\
\hline Black & $3(23.1 \%)$ \\
\hline Hispanic & $1(7.7 \%)$ \\
\hline Asian & $4(30.8 \%)$ \\
\hline Unknown & $4(30.8 \%)$ \\
\hline Total n & 11 \\
\hline \multicolumn{2}{|l|}{ ACR criteria for SLE diagnosis, $n(\%)$} \\
\hline Malar rash & $2(18.2 \%)$ \\
\hline Discoid rash & $3(27.3 \%)$ \\
\hline Photosensitivity & $2(18.2 \%)$ \\
\hline Oral ulcers & $2(18.2 \%)$ \\
\hline Arthritis & $10(90.9 \%)$ \\
\hline Serositis & $6(54.5 \%)$ \\
\hline Renal disorder & $2(18.2 \%)$ \\
\hline Neurological disorder & $4(36.4 \%)$ \\
\hline Haematological disorder & $8(72.7 \%)$ \\
\hline Immunological disorder** & $10(90.9 \%)$ \\
\hline Anti-nuclear antibody (ANA) & $11(100.0 \%)$ \\
\hline
\end{tabular}

SLE: Systemic Lupus Erythematosus, MG: Myasthenia Gravis

ACR: American College of Rheumatology

*SLE duration at time of MG diagnosis

**anti-DNA or anti-Smith, anti-cardiolipin antibodies, or lupus anticoagulant

Table 1. Demographics and clinical characteristics of 13 SLE patients who later developed

Myasthenia Gravis, reported in the literature

The mean age at SLE diagnosis was $25.6 \pm 9.5$ years (6-39 years), with a mean SLE duration (at the time of MG diagnosis) of $8.1 \pm 8.2$ years (a few months to 30 years). The mean age at which the SLE patients developed MG was $33.5 \pm 13.8$ years (12-69 years). As we expected, the majority of the patients were female (84.6\%); 1 patient was white (7.7\%), 3 were black (23.1\%), 1 was Hispanic (7.7\%), 4 were Asian (30.8\%) and 4 unknown (30.8\%). In the 11 out of 13 patients for whom the data were available, the distribution of ACR SLE criteria at any point up to the MG diagnosis was as follows: malar rash $(18.2 \%)$, discoid rash $(27.3 \%)$, photosensitivity $(18.2 \%)$, oral ulcers $(18.2 \%)$, 


\section{European Journal \\ of Case Reports in \\ Internal Medicine}

arthritis (90.9\%), serositis (54.5\%), renal disorder (18.2\%), neurological disorder (36.4\%), haematological disorder (72.7\%), immunological disorder (90.9\%) and ANA positivity (100.0\%).

Calculation of MG incidence in our SLE cohort

From 2004 to 2013, we followed 380 female patients with ACR criteria for SLE for an average of 7.5 years each, yielding 2850 person-years of follow-up. During this time, one case of MG was diagnosed. In comparison, the incidence rate of MG in the female general population is most often quoted as 3 cases $/ 100,000$ person-years.

\section{Discussion}

Myasthenia gravis and systemic lupus erythematosus are autoimmune diseases that, as mentioned in the introduction, have some similarities in terms of demographics, although they are very distinct entities. MG is an organ-specific (neuromuscular junction) autoimmune disease with the highest incidence in women of reproductive age $(<40)$, while SLE is non-organ-specific and also often involves women of reproductive age and beyond ${ }^{1,2,4}$. The literature contains descriptions of both MG developing after SLE and vice versa ${ }^{2,3}$. Still, the development of MG in SLE patients appears to be rather rare.

In this study, a total of 13 cases of MG arising in SLE patients were found through a systematic literature review. Most cases in the literature were relatively young, with a mean SLE duration of 8 years at the time of MG diagnosis, and many were non-white. One article ${ }^{1}$ suggested that SLE patients with MG diagnoses seem to have less frequent cutaneous and renal manifestations than what has been described in SLE patients without MG. Another publication studied 78 MG patients and suggested a much higher prevalence of SLE (six cases, 7.7\%) than in the general population; in that paper, all SLE cases with MG were female.

In summary, the development of MG in SLE is still a rare event. Regardless, in any SLE patients with muscle weakness and characteristic features (e.g. ptosis, muscle fatigability) suggesting MG, neurological evaluation, including AChR antibodies and electrodiagnostic testing, can help exclude or confirm MG.

\section{References}

1. Jallouli M, Saadoun D, Emymard B, Leroux G, Haroche J, Le Thi Huong D, et al. The association of systemic lupus erythematosus and myasthenia gravis: a series of 17 cases, with a special focus on hydroxychloroquine use and a review of the literature. J Neurol 2012;259:1290-1297.

2. Omar HA, Alzahrani MA, Al Bshabshe AA, Assiri A, Shalaby M, Dwefar A, et al. Systemic lupus erythematosus after thymectomy for myasthenia gravis: a case report and review of the literature. Clin Exp Nephrol 2010;14:272-276. 
3. Hrycek A. Systemic lupus erythematosus and myasthenia gravis. Pol Arch Med Wewn 2009;119:582-585.

4. Castrejón I, Shum K, Tseng C-E, Askanase A. Association between myasthenia gravis and systemic lupus erythematosus: three case reports and review of the literature. Scand I Rheumatol 2011;40:486-490. 